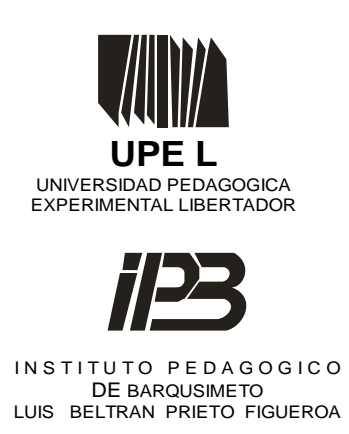

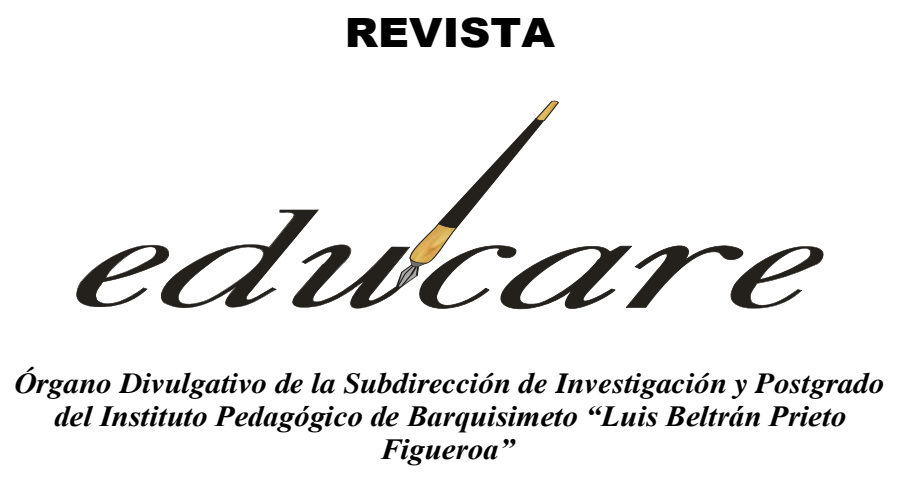

BARQUISIMETO - EDO. LARA - VENEZUELA

NUEVA ETAPA

FORMATO ELECTRÒNICO

DEPOSITO LEGAL:

PPI2O 10O2LA3674

VOLUMEN 19 № 3

ISSN: $2244-7296$

SEPTIEMBRE - DICIEMBRE 2015

\title{
ESTRATEGIAS COGNITIVAS PARA EL FORTALECIMIENTO DEL PENSAMIENTO CRÍTICO EN ESTUDIANTES DE EDUCACIÓN MENCIÓN CIENCIAS SOCIALES
}

COGNITIVE STRATEGIES FOR THE ENHANCENMENT OF CRITICAL THNKING IN TEACHER TRAINEES MAJORING SOCIAL SCIENCES

\author{
GOUVEIA MUÑETÓN, EDITH LUZ* \\ ATENCIO RAMÍREZ, MAXULA* \\ DE NOBREGA, JANETH* \\ "UNIVERSIDAD DEL ZULIA (LUZ)
}

FACULTAD DE HUMANIDADES Y EDUCACIÓN, ESCUELA DE EDUCACIÓN. CENTRO DE ESTUDIOS GEOGRÁFICOS. MARACAIBO, ZULIA 
ESTRATEGIAS COGNITIVAS PARA EL FORTALECIMIENTO DEL PENSAMIENTO CRÍTICO EN ESTUDIANTES DE EDUCACIÓN MENCIÓN CIENCIAS SOCIALES

Edith Gouveia, Maxula Atencio y Janeth de Nobrega (p. 28-51)

\section{ESTRATEGIAS COGNITIVAS PARA EL FORTALECIMIENTO DEL PENSAMIENTO CRÍTICO EN ESTUDIANTES DE EDUCACIÓN MENCIÓN CIENCIAS SOCIALES}

\section{COGNITIVE STRATEGIES FOR THE ENHANCENMENT OF CRITICAL THNKING IN TEACHER TRAINEES MAJORING SOCIAL SCIENCES}

\begin{tabular}{|l|l|}
\hline INVESTIGACIÒN & \multicolumn{1}{|c|}{$\begin{array}{c}\text { Gouveia Muñetón, Edith Luz } \\
\text { Atencio Ramírez, Maxula } * * \\
\text { De Nobrega, Janeth } * * *\end{array}$} \\
LUZ
\end{tabular}

\footnotetext{
${ }^{*}$ Dra. en Ciencias de la Educación. Profesora Titular. Directora del Centro de Estudios Geográficos de la Facultad de Humanidades y Educación de la Universidad del Zulia. edithgouveia@ yahoo.com,

**Dra. en Ciencias Humanas: Profesora Titular. Coordinadora de la Maestría en Geografía. Jefe de Departamento de Historia de la Facultad de Humanidades y Educación de la Universidad del Zulia maxuatencio@gmail.com

***Lcda. en Educación, mención Ciencias Sociales. Actualmente cursa la Maestría en Geografía. Ayudante académica de la maestría.' jancer.power@gmail.com
}

Revista EDUCARE, Volumen 19, Número 3, Septiembre -Diciembre 2015. ISSN: 2244-7296 Página 29 


\section{SITUACIÓN PROBLEMÁTICA}

Las estrategias cognitivas aluden directamente a las distintas capacidades intelectuales de los individuos al hacer algo. Estas estrategias de acuerdo con Hartman y Sternberg (1993), pueden ser numerosas, variadas y de gran utilidad, a la hora de trabajar en las distintas áreas del conocimiento y cuya actividad específica es afectada por múltiples factores que dependen de la materia, de la tarea, de las actitudes y de las variables del contexto donde tienen lugar.

En la misma línea de pensamiento, Elliot (1993) señala que las estrategias cognitivas no son elementos aislados ni independientes, sino que están vinculadas a una estructura, esto implica que el desarrollo de una habilidad determinada no se genera desconectado de los procesos paralelos, con los cuales ocurre el desarrollo de otras habilidades, aunque algunas experiencias se propicien con la intencionalidad de contribuir al desarrollo de una habilidad particular, también implica que para el logro de una determinada competencia se involucra más que una habilidad específica, una estructura de habilidades.

Por ello, son las facilitadoras del conocimiento, debido a que operan directamente sobre la información: recogiendo, analizando, comprendiendo, procesando y guardando información en la memoria, para luego poder recuperarla y utilizarla en el lugar apropiado. Según Zapata (2005) las estrategias cognitivas son las facilitadoras de la cantidad y calidad de conocimiento que se tiene y permiten que el pensamiento humano tenga la capacidad de almacenar información, procesarla y transformarla; para organizarla y dar origen a productos nuevos.

En ese sentido, la Universidad está dirigida a realizar una función rectora de educación, cultura y ciencia, por lo cual sus actividades deben orientarse a crear, asimilar y difundir el saber mediante la investigación, la enseñanza y extensión. Por ello y como necesidad de dar respuesta a los nuevos contextos de aprendizajes que se requieren, se observa en el ámbito educativo la influencia de la revolución cognitiva en los procesos del pensamiento creativo, contribuyendo en gran medida al redimensionamiento o redescubrimiento de la mente humana.

Por lo tanto, la nueva visión de educación implica la definición de novedosas

Revista EDUCARE, Volumen 19, Número 3, Septiembre -Diciembre 2015. ISSN: 2244-7296 Página 30 
estrategias en los modelos que permitan el desarrollo de pensamiento crítico. A tal fin, se requiere de un proceso que combine los aspectos científicos, sociales, culturales y racionales del ser humano, quien por medio de la investigación, está constantemente en la búsqueda de nuevos conocimientos que son producto de su trabajo y experiencia, y le permitirá afrontar el reto de la modernización y globalización con mayores probabilidades de éxito dentro del ámbito universitario.

Además, en el mundo contemporáneo que experimenta una rápida e incesante transformación, se percibe la necesidad de un nuevo modelo de educación universitaria, que debe estar centrada en el estudiante, en consecuencia, requiere una evolución no solo de los contenidos, sino de los métodos y medios de enseñanza y el aprendizaje, que han de estimular el desarrollo del pensamiento crítico y basarse en nuevos tipos de colaboración.

El informe de la UNESCO, (generado por el equipo de Delors, 1996), plantea el reto particular que enfrentan las universidades contemporáneas para poder potenciar en sus discípulos la comprensión, despertar la curiosidad intelectual, estimular el sentido crítico y adquirir al mismo tiempo autonomía de juicio. Además se señala otro elemento clave, "fomentar la cooperación en el aprendizaje". La declaración sobre la educación superior en el siglo XXI, se ha convertido en documento orientador de acción prioritaria para el cambio y el desarrollo de las universidades, en su Artículo 9 dedicado especialmente al pensamiento crítico y creatividad, declara que:

Las instituciones de educación superior según la UNESCO(1998), deben formar a los estudiantes para que se conviertan en ciudadanos informados y motivados, provistos de sentido crítico, capaces de analizar problemas de la sociedad, buscar soluciones, aplicarlas y asumir responsabilidades sociales.

Pero para lograr tal cometido, es necesario que el estudiante desarrolle una serie de habilidades cognitivas que le permitan percibir, asimilar, almacenar y recuperar conocimientos; supone también una destreza mental, que le habilite para desarrollar una tarea. Sin embargo, diversos investigadores Araujo, (2011), Fernández, (2009), Galindo, (2010), Pírela, (2011) y Romero, (2012), permiten sustentar que un alto porcentaje de los estudiantes que ingresan al sistema de Educación universitaria y secundaria presentan deficiencias para razonar a nivel de operaciones formales y para pensar en forma crítica y

Revista EDUCARE, Volumen 19, Número 3, Septiembre -Diciembre 2015. ISSN: 2244-7296 Página 31 
creativa.

En ese contexto, es de destacar que si bien los estudiantes presentan dificultades de razonamiento, poco se ha hecho desde la educación para favorecer el desarrollo de habilidades cognitivas, pues no les enseñan a resolver problemas. Los docentes confían en que sus estudiantes, discentes aprenden a resolver situaciones problemáticas por observación. Además, piensan que los atributos y el juicio de los estudiantes son suficientes.

Tal situación contradice lo establecido en todas las leyes que rigen el sistema educativo y que señalan la importancia de fomentar las capacidades críticas en los estudiantes. Concretamente la Ley Orgánica de Educación (LOE, 2009) señala en los objetivos de cada una de las etapas educativas, primaria y secundaria, el fomento del "sentido crítico". En el contexto universitario, algunas de las competencias transversales señaladas son la capacidad de análisis y síntesis, la habilidad de gestión de información, la resolución de problemas, la toma de decisiones, la capacidad crítica, entre otras., todas ellas habilidades del pensamiento crítico.

Considerando las aseveraciones anteriores, se hace necesario destacar la situación particular que presentan los estudiantes de educación de la mención Ciencias Sociales en quienes se percibe falta de estrategias cognitivas así como de pensamiento crítico necesarios para un exitoso desenvolvimiento académico y profesional. Los mencionados estudiantes, presentan una falta de repertorio adecuado para manejar los conceptos y conocimientos relacionados con el Área de Ciencias Sociales. De igual manera, la falta de motivación al logro que obstaculiza la calidad de los procesos cognitivos de los estudiantes en cuestión, ya que no se entusiasman en la búsqueda de los conocimientos y habilidades que requieren para abordar con seguridad y confianza el análisis de problemas y su resolución. Estas observaciones, coincide con los resultados encontrados por Romero, (2012) Araujo (2011), Pírela (2011), Fernández (2009), quienes señalan que esa práctica pedagógica es cuestionada por su acento tradicional pues se aferra a una labor formativa empeñada en fijar nociones y conceptos geográficos a través del dictado, la copia, el dibujo y el calcado. Es una enseñanza geográfica descriptiva, determinista y enciclopedista, contradictoria con los avances de la disciplina geográfica más preocupada por las 
situaciones ambientales y sociales que emergen de la organización del espacio por los grupos humanos.

Como consecuencia de lo antes expuesto, se pone de manifiesto ciertas deficiencias en la aplicación de procesos mentales superiores tales como análisis, síntesis e interpretación y, como resultado de esto, se plantea un proceso de aprendizaje tardío y tedioso donde el estudiante no hace ningún esfuerzo de tipo cognitivo debido a carencias de habilidades de pensamiento crítico. En contraste con el planteamiento anterior, Romero, (2012) Araujo (2011), Pírela (2011), Fernández (2009),señalan que los estudiantes típicamente rinde en preguntas de repetir información y de utilizar habilidades básica (observación, descripción, comparación) pero rinden mal en preguntas que requieren de análisis más profundos. Por este motivo, surgió la inquietud de investigar sobre las estrategias cognitivas y el desarrollo del pensamiento crítico en estudiantes de la carrera de Educación mención Ciencias Sociales de la Facultad de Humanidades y Educación de la Universidad del Zulia.

En el marco de la problemática anteriormente descrita surge la siguiente interrogante: ¿Cuáles estrategias cognitivas contribuyen al desarrollo del pensamiento crítico en estudiantes de la carrera de Educación Mención Ciencias Sociales de la Facultad de Humanidades y Educación de LUZ? y como objetivo de la misma se propuso determinar estrategias cognitivas para el desarrollo del pensamiento crítico en estudiantes de la carrera de Educación Mención Ciencias Sociales de la Facultad de Humanidades y Educación de LUZ.

\section{FUNDAMENTACIÓN CONCEPTUAL}

\section{Estrategias cognitivas}

En la práctica educativa seleccionar y desarrollar las estrategias de enseñanza es un papel medular, ya que se delinea el proceso de intervención del docente y de los estudiantes. Una estrategia didáctica es un plan, procedimientos o actividades secuenciadas que orientan el desarrollo de las acciones del maestro, estudiantes y que conducen al logro de un objetivo. El trabajo de los docentes consiste en planear la enseñanza de tal manera que los estudiantes puedan usar una o más estrategias cognitivas

Revista EDUCARE, Volumen 19, Número 3, Septiembre -Diciembre 2015. ISSN: 2244-7296 Página 33 
para aprender el material, para procesar activamente el contenido. Son las que ayudan a los estudiantes a lograr las metas relativas a la construcción del conocimiento. En términos de Gaskins y Elliot (1999:64), "son los procesos de pensamiento que se emplean para hacer el trabajo concreto del pensamiento". Ambas estrategias, organizativas y cognitivas, son necesarias, se complementan y generalmente se emplean en forma simultánea durante la enseñanza.

Se puede decir entonces, que las estrategias cognitivas son un conjunto de operaciones mentales, cuyo objetivo es que el individuo integre la información adquirida a través de los sentidos, en una estructura de conocimiento que tenga sentido para él. El concepto de habilidad cognitiva, de acuerdo a Chadwick y Rivera (1991), es una idea de la Psicología Cognitiva que enfatiza que el sujeto no sólo adquiere los contenidos mismos sino que también aprende el proceso que usó para hacerlo: aprende no solamente lo que aprendió sino como lo aprendió.

Por su parte Rigney (1978), define las estrategias cognitivas son operaciones y procedimientos que el individuo emplea para adquirir, retener y recuperar diferentes tipos de conocimientos y ejecución; suponen en el estudiante capacidades de representación, como lectura, imágenes, habla, escritura y dibujo; capacidades de selección, entre las que destacan atención e intención; y capacidades de autodirección, tales como auto programación y autocontrol.

Igualmente, Gaskins, y Elliot (1999:97) señalan unos ejemplos de estrategias cognitivas, tal como se expone a continuación: Explorar el texto o material, leer el título, mirar los dibujos, a fin de formar un esquema mental, acceder al conocimiento previo, recordar, predecir, formular hipótesis y/o plantear objetivos, comparar la nueva información con lo que se sabe, crear imágenes mentales de lo que se está procesando, hacer inferencias, generar preguntas y pedir aclaraciones, seleccionar ideas importantes, elaborar pensando ejemplos, contra-ejemplos, analogías, comparaciones, parafrasear o resumir para representar la sustancia de la información, evaluar ideas presentadas en el texto, pensar críticamente sobre el objetivo y el punto de vista del autor, incluyendo si las afirmaciones y hallazgos están o no apoyados por pruebas, monitorear el avance/logro de objetivos, clasificar información sobre la base de atributos, identificar relaciones, modelos 
ESTRATEGIAS COGNITIVAS PARA EL FORTALECIMIENTO DEL PENSAMIENTO CRÍTICO EN ESTUDIANTES DE EDUCACIÓN MENCIÓN CIENCIAS SOCIALES

Edith Gouveia, Maxula Atencio y Janeth de Nobrega (p. 28-51)

(de causa/efecto, orden temporal, todo/parte), organizar ideas claves en gráficos, esquemas, listas.

\section{Pensamiento crítico}

Paul y Elder (2004) esbozan que el pensamiento crítico es ese modo de pensar sobre cualquier tema, contenido o problema- en el cual el pensante mejora la calidad de su pensamiento al apoderarse de las estructuras inherentes del acto de pensar y al someterlas a estándares intelectuales; a fin de cuentas, se trata, de formar un pensamiento crítico.

Por su parte, Beltrán (1996) concibe el pensamiento crítico como una estrategia de aprendizaje que forma parte de los procesos de personalización, junto al pensamiento creativo. El pensamiento crítico, como toda estrategia de aprendizaje, se desarrolla a través de una serie de técnicas dentro de las que destacan las siguientes: detección de información sesgada, discusión socrática, controversia y análisis de experiencias. Ahora bien, dichas técnicas se apoyan en el modelo de instrucción de estrategias de

Otra interesante definición es la propuesta por Ennis y Norris en (1989), quienes plantean que pensar críticamente es "decidir razonable y reflexivamente acerca de qué creer o hacer".

Adicionalmente, una definición de Paul y Elder (2004) plantea que "el pensamiento crítico se entiende mejor como la habilidad de los sujetos para hacerse cargo de su propio pensamiento. Esto requiere que desarrollen criterios y estándares apropiados para analizar y evaluar su propio pensamiento y utilizar rutinariamente esos criterios y estándares para mejorar su calidad.

\section{Fases o niveles del pensamiento crítico}

El pensamiento crítico se fundamenta en la psicología cognitiva que se ocupa de los procesos a través de los cuales el individuo obtiene conocimientos, estas fases o niveles así llamadas por Paul y Elder (2004), se dividen en tres: Literal, inferencial y crítico, desarrollándose en cada uno de ellos una serie de capacidades específicas que muestran claramente la ruta a seguir hasta llegar al nivel, más alto se los procesos de pensamiento.

Revista EDUCARE, Volumen 19, Número 3, Septiembre -Diciembre 2015. ISSN: 2244-7296 Página 35 
I. Nivel literal:

1. Percepción. 2. Observación. 3. Discriminación. 4. Nombrar o Identificar. 5. Emparejar. 6. Secuencia u ordenador.

II. Nivel inferencia:

1. Inferir. 2. Comparar-contrastar. 3. Categorizar-clasificar. 4. Describir-explicar.

5. Analizar. 6. Indican causas y efectos. 7. Interpretar. 8. Resumir-sintetizar. 9.

Predecir-estimar. 10. Generalizar. 11. Resolución de problemas.

III. Nivel crítico:

1. Debatir-argumentar: 2. Evaluar-juzgar y criticar

\section{Ventajas del pensamiento crítico}

Los beneficios del pensamiento crítico para la persona son muchos, Paul y Elder (2004) destacan las características de quien se ejercita en el pensamiento crítico: tiene agudeza perceptiva, hace cuestionamientos permanentes, construye y reconstruye saberes, es de mente abierta, posee una valoración justa, tiene control emotivo y coraje intelectual. Otras bondades para el educando son que "permitirá brindar información, definir, formular hipótesis y resolver problemas, evaluar las pruebas aplicadas y obtener conclusiones". En otras palabras, los estudiantes "serán individuos autosuficientes y responsables para enfrentar las demandas en este mundo en permanente cambio e incertidumbre".

\section{Principales estrategias y técnicas específicas para desarrollar el pensamiento crítico}

Paredes (2006:65) establece algunas técnicas a emplear para el desarrollo del pensamiento crítico:

"Iniciar y desarrollar el planteamiento de preguntas, ayudar a los estudiantes a desarrollar la capacidad de utilizar diversas fuentes de información, enseñar una metodología de investigación, establecer discusiones en clase, legitimar la búsqueda, es decir, apoyar y aprobar discusiones abiertas, animar a los estudiantes a reflexionar respecto a sus propias experiencias, otorgar un nuevo papel al docente, considerar el aspecto afectivo, realizar propuestas y alternativas de solución, elaborar conclusiones, elaborar un informe sobre el tema investigado" 


\section{METODOLOGÍA}

La investigación se realizó desde una perspectiva positivista, se asumió el carácter descriptivo, de campo, bajo un diseño no experimental, transeccional, pues el estudio recolectó y describió las opiniones reveladas por los estudiantes, tal y como se manifestaron en el momento de la aplicación del cuestionario. (Chávez 2004). Se realizó un arqueo bibliográfico para obtener y explicar los fundamentos conceptuales y metodológicos sobre las estrategias cognitivas y el pensamiento crítico. La población estuvo conformada por 111 estudiantes de la carrera de Educación Mención Ciencias Sociales de la Facultad de Humanidades y Educación de Luz, de la Unidad curricular Didáctica de la Ciencias Sociales, del segundo periodo del 2013. La recolección de datos se efectuó mediante un cuestionario, con cinco opciones de respuestas ( siempre, casi siempre, a veces, casi nunca, nunca.) y 32 itemes. El instrumento fue validado por el juicio de siete expertos, y su confiabilidad se calculó por el coeficiente Alfa Cronbach, con resultados altamente confiables de 0,90. Para el análisis de datos se construyó un baremo de interpretación con los siguientes intervalos y categorías (3,27 - 4 alto dominio, 2,51 - 3,26moderado dominio, 1,76 - 2,50 bajo dominio y 1 - 1,75 muy bajo dominio) de las estrategias y del pensamiento crítico, para ubicar los puntajes arrojados por los instrumentos y la estadística descriptiva para el análisis de los resultados.

\section{RESULTADOS DE LA INVESTIGACIÓN}

\section{Análisis de los datos}

Las variables objeto de investigación se agruparon en estrategias cognitivas y pensamiento crítico a través de las dimensiones: Estrategias cognitivas en estudiantes y características del pensamiento crítico, medidas a través de un cuestionario de múltiples respuestas direccionado a los estudiantes de la mención Ciencias Sociales.

Se obtuvieron los siguientes resultados representados en un primer término por las frecuencias y porcentajes de los indicadores, y en segundo, el cálculo de las medidas de tendencia central a través de los promedios por indicador y dimensiones respectivamente, (medias y medianas) para luego, establecer el comportamiento de las variables.

En cuanto a la dimensión Estrategia cognitiva, la frecuencia obtenida en las 
respuestas para los itemes 1 y 2 dirigidos a medir el indicador explora el material; a tal respecto, se observa al mayor porcentaje, esto es, un 50,45\% de los estudiantes se ubicó en la alternativa 4, correspondiente a Casi Siempre, seguido de un 31,53\% en la alternativa 3 , es decir A Veces, revelando que los estudiantes al momento de usar procedimientos para adquirir, retener y recuperar el conocimientos, explorar el texto o material, leer el título, mirar los dibujos, a fin de formar un esquema mental.

Los porcentajes más bajos se reflejaron en la alternativa 2 Casi Nunca con 16,22 y 1,80\% Siempre. Esta situación también se evidenció en el análisis de los resultados efectuados por las autoras, cuando advierte que en la medias aritméticas de 3,38; esta cifra, que al ser ubicada en el baremo de interpretación, se coloca en la categoría de Moderado Dominio de las estrategias cognitivas, lo cual significa que los estudiantes moderadamente, exploran el material a utilizar para la obtención del conocimiento.

Para el indicador: Acceder al conocimiento previo, la información respecto a los itemes 3 y 4. De acuerdo a ello, el 47,75\% de los estudiantes encuestados optaron por la alternativa 3 A Veces, seguido de un 38,74\% en la categoría casi siempre; sin embargo, un porcentaje considerable de ellos, el 9,91\%, se ubicó en la alternativa 2 Casi Nunca.

Adicionalmente ser resalta que un 3,60 escogió la alternativa Siempre esto indica que los estudiantes recuerdan lo que se conoce sobre los contenidos, temas desarrollados durante el procesos de exploración que realizan los docentes al momento de iniciar un contenido en particular, solo algunos manifestaron Casi Nunca recordar al momento que el docente comienza a abordar el tema con las técnicas de la pregunta y respuesta o la lluvia de ideas.

También, Al ubicar la media en el baremo respectivo, se observa que ésta se colocó por encima de la mediana en la categoría moderado expresado en los promedios de 3,36, lo que evidencia que los estudiantes medianamente recuerdan lo que conocen sobre las preguntas, itemes, contenidos o temas abordados por los docentes al momento de iniciar una clase, motivo por el cual se resalta en este estudio la participación de los estudiantes en el momento de entregar las respuestas concretas ante un tema determinado.

En cuanto al indicador predecir, se puede ver que los mayores puntajes, para los estudiantes, se ubicaron en las alternativas A Veces, Casi Siempre y Casi Nunca con 
$35,14 \%, 32,43$ y 27,93 respectivamente, indicando con ello que, según su propia perspectiva, el estudiante supone qué información se presentará, basándose en el marco mental desarrollado mientras se examinaba el material y en el conocimiento anterior al momento de generar el conocimiento. Al igual que el indicador anterior, los puntajes para éste se ubicaron en la categoría de Moderado con promedios de 2,95, indicando que los estudiantes presumen la información que se mostrará en clase, o al momento de estudiar o aprender.

Se exponen los resultados para el indicador comparar la nueva información con lo que se sabe, cuyo mayor puntaje para los estudiantes es el siguiente: un 54,05\%, seleccionó la opción A Veces, seguido de un 20,72 en la opción Casi Siempre, en tanto que el 15,32 se ubicaron en Siempre. Los menores valores se reflejaron en la alternativa Casi Nunca con 7,21 y 2,70 eligió Nunca

Esto indica que los estudiantes de la mención utilizan estrategias Casi Siempre o A Veces destinadas a crear o potenciar enlaces adecuados entre los conocimientos previos y la información nueva que ha de aprenderse, asegurando con ello una mayor significatividad de los aprendizajes logrados. Al contrastar los promedios con el baremo de interpretación, los mismos se encuentran en la categoría Alto Dominio de las estrategias cognitivas y pensamiento crítico con 3,39 reflejando así los encuestados estar de acuerdo en que vinculan los conocimientos previos con la información nueva.

Los resultados para el indicador Crear imágenes mentales de lo que se está procesando, medido mediante el ítem 7; se encontró que el 40,54 de los estudiantes eligieron la alternativa Casi Nunca, un 37,84 eligió la alternativa A Veces y el 19,82 Nunca, indicando así que los estudiantes no alcanzan hacer cuadros mentales para poder visualizar lo que se está procesando en el proceso de aprendizaje. El porcentaje más bajo se colocó en $1,80 \%$ que respondió a la alternativa Casi siempre. Para este indicador, el promedio se ubicó en la categoría de Bajo Dominio de las estrategias cognitivas y pensamiento crítico, de acuerdo al baremo construido para tal fin.

La información para el indicador Hacer inferencias, medido a través del ítem 8, en los estudiantes, la selección fue en un 33,59\% para la alternativa 3 (A Veces) y 2 (Casi Nunca) con 40,54\% y 13,51\% eligió la alternativa Nunca. Tales resultados demuestra que,

Revista EDUCARE, Volumen 19, Número 3, Septiembre -Diciembre 2015. ISSN: 2244-7296 Página 39 
a juicio de los propios estudiantes, no almacenan hechos, datos sobre la información que se enseñó y lo ordena con información o creencias, opiniones que posee para sacar conclusiones. Al aplicar la media y ubicarla en el baremo respectivo, se observa que éstas se colocaron 2,38; es decir, en la categoría Bajo Dominio de las estrategias cognitivas.

Las respuestas para el indicador Generar preguntas y pedir aclaraciones correspondiente al ítem 9 se ubicaron en $47,75 \%$ la alternativa 3 y el 27,03\% la alternativa 2; mientras que $19,82 \%$ la alternativa Casi Siempre (4), seguido de un3,60\% en la alternativa Siempre.

Tales resultados indican que la población encuestada al momento de esbozar preguntas en relación de la información, tema o contenido enseñado por el docente, los estudiantes no generan interrogantes de manera asertiva sino esperan que se respondan a medida que avanza en la presentación. Al contrastar los promedios con la mediana de distribución en el respectivo baremo, se destaca que los estudiantes $(2,96)$ se encuentran en la categoría Moderada, indicativo que las acciones para que el estudiante aborde la información a través de las preguntas y pedir aclaraciones están presentes en una moderada proporción.

Para el indicador Seleccionar ideas importantes con los itemes10 y 11, notándose que un 42,79\% de los encuestados optó por la opción 3 (A Veces), y un 39,64\% en la alternativa 2 (Casi Nunca). De esta manera, se visualiza que a opinión de los estudiantes que a ellos les dificultad Identificar los tópicos más importantes de los párrafos en texto y enunciar en las propias palabras la idea más importante presentada. Al ubicar los promedios obtenidos para este indicador, es decir, 2,51 en el baremo de interpretación, se observa que se encuentran en un bajo dominio de las estrategias cognitivas.

Los resultados para el indicador elaborar pensando, con los itemes 12 y 13, donde los mayores puntajes se ubicaron en las alternativas A Veces y Casi Nunca con 48,65\% y $27,48 \%$, seguidos por un $13,06 \%$ en la categoría Nunca y con $8,11 \%$ para Casi Siempre. Es decir, los estudiantes encuestados no creen en su capacidad para pensar en qué sentido la información es similar a información que se conoce (situaciones) o advertir en qué sentido es diferente de una situación similar. Los promedios para este indicador se ubican en la categoría de Moderado Dominio de las estrategias cognitivas con promedios de 2,60.

Revista EDUCARE, Volumen 19, Número 3, Septiembre -Diciembre 2015. ISSN: 2244-7296 Página 40 
El indicador Evaluar ideas presentadas en el texto, los cuales indican que el 50,90\% y 27,48\% de los estudiantes seleccionaron las opción 3 (A Veces) y 2 (Casi Nunca) respectivamente. Por su parte, el 16,22\% señalaron la alternativa 1 (Nunca) y el 5,41\% Casi Siempre. Los datos obtenidos, permiten inferir que para el estudiante pensar críticamente sobre el objetivo y el punto de vista del autor, incluyendo si las afirmaciones y hallazgos están o no apoyados por pruebas, no es clave para la obtención del aprendizaje. Al ubicar los promedios de estos valores en el baremo, los mismos se encuentran 2,45; sin embargo, todos se ubican en categoría Bajo Dominio de las estrategias cognitivas.

En relación a la dimensión Estrategias cognitivas, puede observarse que respecto al parafrasear o resumir, el 46,85\% de los estudiantes, respondieron a la alternativa 3 (A Veces), seguido de un 39,19\% en la alternativa 2, (Casi Nunca); por otro lado, con un 9,91\% y 3,60\% se ubicaron en las Nunca y Casi Siempre respectivamente.

El porcentaje más bajos se reflejó en $0,4 \%$ en la alternativa 1 (Siempre). Se evidencia que los estudiantes al momento de enunciar o manifestar a fondo del material, contenido o tema tratado con sus propias palabras, omitiendo ideas repetitivas A Veces o Casi Nunca lo hacen. Tanto el indicador 16 y 17 se encuentran con una mediana de 2,45, es decir, Bajo Dominio de las estrategias cognitivas,indicativo que el estudiante no parafrasea al momento de obtener el aprendizaje.

En referencia al indicador Clasificar información sobre la base de atributos. Un 40,54\% de los encuestados optaron por la alternativa 2 (Casi Nunca), seguido de un 36,49\% en la alternativa 3 (A Veces), mientras con un $14,41 \%$, y un $8,11 \%$ señalaron que Nunca y Casi Siempre. Estos datos muestran que desde la perspectiva de los estudiantes al momento de identificar brechas en el conocimiento o la comprensión, ampliar el aprendizaje a nuevas preguntas, rellenar las brechas, ellos adoptan una acción indiferente. Asimismo, el promedio de 2,40 arrojados para este indicador, al ser contrastados con el baremo de interpretación, se encuentran en bajo dominio de las estrategias cognitivas.

El indicador Clasificar información, fue medido a través del ítem 20. A tal respecto, se observa que 38,74\% de la población seleccionó la alternativa 3 (A Veces), seguido de $29,73 \%$ en Casi Nunca. Por otro lado, el 26,13\% eligieron las alternativas Nunca y 3,60\% Casi Siempre, indicando que al momento enumerar acontecimientos importantes, nombres, 
fechas, lugares a medida que se presenta la información y luego vincularlo con otras ideas.

De tal manera, se advierte en la presente investigación que los estudiantes A Veces o Casi Nunca lo hacen y por otro lado no los clasifican para retenerlos, comprender los conceptos, hechos, datos. Al aplicar la media y ubicarla con el baremo respectivo, se observa que ésta se encuentra por debajo de la mediana en la categoría Bajo Dominio con promedios de 2,25 .

En cuanto al indicador Identificar relaciones y modelos, visualizado en la tabla 14, los mayores puntajes recaen en la alternativa Casi Nunca con 58,56\% y 18,02 en A Veces. Mientras que $14,41 \%$ en Casi Siempre y 9,01\% Nunca. En relación al indicador los estudiantes Casi Nunca identifican relaciones de causa/efecto y de orden temporal. También es importante destacar en el presente estudio, que este indicador se ubicó en el respectivo baremo en la categoría de Bajo Dominio de las estrategias cognitivas, con promedio de 2,38.

Para el indicador Organizar ideas claves, donde se destaca al 49,55\% de los encuestados seleccionaron la alternativa 3 (A Veces) y 33,33\% casi nunca. El 9,01\% seleccionaron la opción 4 (Casi Siempre) seguido por 7,21\% en nunca. Se evidencia en este indicador que, según su opinión, el estudiante A Veces usa estrategias que le permiten $\begin{array}{lllll}\text { organizar } & \text { a } & \text { la nformación nueva }\end{array}$ que se aprenderá. Al aplicar la media y contrastar la con el baremo respectivo, se observa, ubicarse por encima de la mediana en la categoría Moderado con promedios de 2,63.

En cuanto al indicador Transferir o aplicar conceptos, el mayor porcentaje 53,15\% se ubica en la alternativa 3 (A Veces), el 24,32\% (Casi Nunca), opinando que casi no se preocupan por aplicar los conceptos aprendidos o por adquirir nueva información relevante, organizándola de manera tal, pueda ser utilizada para futuras aplicaciones. Para este indicador los promedios con respecto al baremo de interpretación con 3,05; entra en la categoría de Moderado. Una vez analizados los dieciséis indicadores que conforman la dimensión estrategias cognitivas, se presenta la Tabla 17, la cual resume los promedios por los indicadores y dimensión.

Como puede evidenciarse, para la dimensión Estrategias Cognitivas se obtuvo un promedio de 2,71 ubicado en la categoría Moderado, indicando que los estudiantes de la 
carrera educación mención Ciencias Sociales, específicamente en la unidad curricular Didáctica de las Ciencias Sociales de la Universidad del Zulia poseen moderadamente el manejo de dichas estrategia tales como operaciones y procedimientos que usa el individuo para adquirir el conocimiento, lo cual permite afirmar que no existe un manejo total de tales procedimientos; es decir, aquellas características requeridas para fortalecer el pensamiento crítico.

En relación con la dimensión característica del pensamiento crítico, para el indicador Agudeza perceptiva, se observa, que 34,68\% de los estudiantes escogió la alternativa A Veces y $33,78 \%$ casi siempre. Igualmente señalaron con $31,08 \%$ la alternativa 2 Casi Nunca y siempre con $0,45 \%$. Se expone así la opinión de los estudiantes al saber que es una potencialidad que permite observar los mínimos detalles de un objeto o tema y que posibilita una postura adecuada frente a los demás. El promedio para este indicador se ubica en el baremo Moderado Dominio $(2,51$ - 3,26) del pensamiento crítico.

Para el indicador Cuestionamiento, el cual se refiere a la disposición para juzgar las situaciones que se le presenten, la frecuencia en las respuestas, destacando que los estudiantes en un porcentaje de 40,54\% respondieron con la alternativa 2 (Casi Nunca), seguido de un $23,42 \%$ en la alternativa 3 (A Veces). Por su parte, señalaron que Casi Siempre y Nunca con un porcentaje igual de $18,02 \%$, coinciden con sus apreciaciones.

El promedio arrojado para este indicador de 2,41al ser contrastado con el baremo se encontró bajo dominio del pensamiento crítico, lo cual significa que ellos no adecuadamente el cuestionamiento de ideas.

Simultáneamente para la dimensión Características del pensamiento crítico, la frecuencia obtenida en las respuestas para los itemes 27 y 28 dirigidos a medir el indicador Construcción y reconstrucción del saber, se observa al mayor porcentaje, con 41,44\% en la alternativa 2, correspondiente a casi nunca, seguido de un $29,72 \%$ en la alternativa 4 Casi Siempre y A Veces con 25,67\%,

Esto deja ver que los estudiantes Casi Nunca están en la capacidad de estar alertas frente a nuevos contenidos, temas. Los porcentajes más bajos se reflejaron en la alternativa 5 Siempre y Nunca con 2,70 y $0,45 \%$. Esta situación también se evidenció en la medias aritméticas de 2,93 que al ser ubicadas en el baremo de interpretación, se colocaron en la

Revista EDUCARE, Volumen 19, Número 3, Septiembre -Diciembre 2015. ISSN: 2244-7296 Página 43 
categoría de Moderado Dominio del pensamiento crítico.

Para el indicador: mente abierta, la información respecto al ítem 29. De acuerdo a ello, el 47,75\% de los estudiantes encuestados optaron por la alternativa 4 (Casi Siempre), seguido de un $28,83 \%$ en la categoría Casi Nunca; sin embargo, un porcentaje considerable de ellos, el 21,62\%, se ubicó en la alternativa 3 A Veces y un 0,90 Siempre y Nunca esto indica que los estudiantes en su mayoría están en la disposición para aceptar las ideas y concepciones además, aunque estén equivocadas. Al ubicar la media en el baremo respectivo, se observa que ésta se colocó en la categoría Moderado Dominio del pensamiento crítico expresado promedios de 3,19.

En cuanto al indicador coraje intelectual, se puede ver que los mayores puntajes, para los estudiantes, se ubicaron en las alternativas A Veces, casi siempre y Casi Nunca con $40,09 \%, 28,38$ y 24,77 respectivamente, indicando con ello que el individuo debe afrontar con entereza y decisión las situaciones difíciles y exponer con altura nuestros planteamientos. Se advierte en la presente investigación, que al igual que el indicador anterior, los puntajes para éste se ubicaron en la categoría de moderado con promedios de 3,06 indicando que los estudiantes A Veces confrontan situaciones difíciles.

Los resultados para el indicador autorregulación, el mayor puntaje para los estudiantes es el siguiente: un 56,30\%, seleccionó la opción A Veces, seguido de un 24,77 enla opción Casi siempre, en tanto que el 13,51 se ubicaron en casi nunca. El menor valor se reflejó en la alternativa siempre con 5,40. Esto indica que los estudiantes de la mención A Veces controlan su forma de pensar y actuar. Al contrastar los promedios con el baremo de interpretación, los mismos se encuentran en la categoría moderado dominio del pensamiento crítico con 3,22 .

Los resultados para el indicador Control emotivo, medido mediante el itemes 34; en ese sentido, se encontró que el 36,93\% de los estudiantes eligieron la alternativa Casi nunca, un 29,72\% eligió la alternativa A Veces y el 27,02 \% casi siempre Nunca, indicando así que los estudiantes no alcanzan a mantener la calma y recordar que se cuestionan las ideas mas no a las personas. Para este indicador, el promedio se ubicó en la categoría de moderado dominio del pensamiento crítico.

La información para el indicador valoración justa, medido a través de los itemes 35 y

Revista EDUCARE, Volumen 19, Número 3, Septiembre -Diciembre 2015. ISSN: 2244-7296 Página 44 
36 en cuanto a las respuestas de los estudiantes la selección fue en un 45,05\% para la alternativa 3 ( A Veces) y 2 (Casi Nunca) con 39,64\% y 9,01\% eligió la alternativa casi siempre, mientras que 4,95\% Nunca. Tales resultados demuestran que, a juicio de los propios estudiantes, A Veces y Casi Nunca se asume una posición objetiva frente a circunstancias donde los sentimientos y las emociones juegan un papel fundamental. Al aplicar la media y ubicarla en el baremo respectivo, se observa que éstas se colocaron $\mathbf{2 , 6 2}$; es decir, en la categoría moderado dominio del pensamiento crítico.

De los resultados analizados anteriormente, y de acuerdo a la dimensión Características del pensamiento crítico presentó un promedio de 2,92 ubicado en la categoría Moderado, de acuerdo con el baremo, evidenciando en el pensamiento crítico de los estudiantes un dominio de moderado. Una vez, finalizado este análisis, se verifica el comportamiento de las variables, producto del promedio de las dimensiones comparado con la mediana en el baremo de interpretación en los siguientes términos: para la dimensión Estrategias cognitivas la media fue de 2,71 y para pensamiento crítico fue de 2,92, indicando este resultado que los estudiante de la carrera educación mención Ciencias Sociales de la Universidad del Zulia, en relación con las manifestaciones de sus dos dimensiones, obtuvo una media aritmética de 2,81 expresando así una manifestación moderada en cuanto al dominio de ambas variables.

\section{Discusión de los Resultados}

En cuanto al objetivo de esta investigación, referido a determinar las estrategias cognitivas para el desarrollo del pensamiento crítico en estudiantes de la carrera de Educación Mención Ciencias Sociales de la Facultad de Humanidades y Educación de LUZ y sobre la base de los resultados obtenidos, con relación a la dimensión estrategias cognitivas empleadas por los estudiantes se obtuvo un promedio de 2,71 observándose de acuerdo al baremo(3,27 - 4 alto dominio, 2,51 - 3,26moderado dominio, 1,76 - 2,50 bajo dominio y 1 - 1,75 muy bajo dominio) que se ubica en la categoría Moderado.

Esto indica que poseen moderadamente el manejo de las estrategias, es decir, aquellos procedimientos, actividades, acciones o procesos mentales que favorecen el aprendizaje, introducen ayudas para pensar y fortalecer el pensamiento crítico. Igualmente hay que

Revista EDUCARE, Volumen 19, Número 3, Septiembre -Diciembre 2015. ISSN: 2244-7296 Página 45 
destacar que los estudiantes, en su mayoría se ubicaron en la alternativa de respuesta A Veces, indicando, que poseen una baja autocritica y reflexión acerca de las habilidades que poseen impidiendo, mejorarlas.

Los resultados expuestos son similares a los hallados por Galindo, (2010)quien en su trabajo de investigación "Estrategias cognoscitivas para la enseñanza y aprendizaje de la nomenclatura y formulación de compuestos inorgánicos”, demostró que los estudiantes no poseen un uso adecuado de las estrategias cognoscitivas y no emplean la mayoría de ellas como es el caso de las estrategias de estudio y ayudas anexas (tomar notas, subrayar, preguntas anexas), las estrategias de organización (mapas mentales, mapas conceptuales, y esquemas).

Ahora bien, tales resultados son relevantes dada la importancia que tienen las estrategias cognitivas para cualquier estudiante. Para Paúl y Elder (2004), ellas son las facilitadoras del conocimiento, operan directamente sobre la información: recogiendo, analizando, comprendiendo, procesando y guardando información en la memoria, para, posteriormente, poder recuperarla y utilizarla dónde, cuándo y cómo convenga.

El trabajo de los docentes consiste en planear la enseñanza de tal manera que los estudiantes puedan usar una o más estrategias cognitivas para aprender el material, para procesar activamente el contenido. Son las que ayudan a los estudiantes a lograr las metas relativas a la construcción del conocimiento. En términos de Gaskins y Elliot (1999), "son los procesos de pensamiento que se emplean para hacer el trabajo concreto del pensamiento". Ella permiten incorporar, comprender y aplicar la información con la finalidad de imprimirle significado, las cuales, son: observar, analizar, ordenar, clasificar, representa, memorizar, interpretar y evaluar.

La importancia de estas estrategias cognitivas es tal, que coincidiendo con Sánchez (1997) facultan al individuo para evaluar y mejorar sistemáticamente lo que piensa y hace. Esto significa, que además de entrenarse para pensar, debe estar al tanto de los procesos estudiados para analizar los pasos que va realizando cuando piensa o cuando realiza alguna tarea intelectual, comparar lo que desea lograr con los productos que obtiene, identificar errores y buscar alternativas para mejorar cada vez más su desempeño, ejecutar procesos de razonamiento, controlar la calidad de lo que piensa y hace, ejercer la crítica 
constructiva, actuar con apertura y comprenderse a sí mismo

En consecuencia, el hecho de que no se encontrara un alto dominio de estas estrategias, supone la posibilidad de mejorarlas a través de determinar estrategias cognitivas para el desarrollo del pensamiento crítico en estudiantes de la carrera de Educación Mención Ciencias Sociales de la Facultad de Humanidades y Educación de LUZ.

Con respecto a las características del pensamiento crítico, los estudiantes de la licenciatura en Educación, mención Ciencias Sociales, revelan un promedio de 2,92 con categoría moderada, demostrando que poseen un dominio moderado de las mismas. Es de señalar, que el pensamiento crítico supone una actividad creativa, se caracteriza por manejar, dominar las ideas. Su principal función no es generar ideas sino revisarlas, evaluarlas y repasar que es lo que se entiende, se procesa, se comunica mediante los otros tipos de pensamiento (verbal, matemático, lógico).

De tal forma, que el pensamiento crítico, según Romero, (2012)supone el proceso intelectualmente disciplinado de conceptualizar, aplicar, analizar, sintetizar y/o evaluar, de manera activa y diestra, información reunida de, o generada por, la experiencia, reflexión, razonamiento o comunicación, como guía para la creencia y la acción. De ello se deduce, que lo característico del pensamiento crítico es que se trata de un pensamiento orientado a la comprensión de problemas, la evaluación de alternativas, la decisión y resolución de los mismos. Pensamiento crítico tiene que ver con comprender, evaluar y resolver.

Según los valores obtenidos, se interpreta que existe un moderado nivel de identificación de agudeza perceptiva, cuestionamiento permanente, construcción y reconstrucción del saber, mente abierta, coraje intelectual, autorregulación, control emotivo y valoración justa lo cual conduce a un bajo nivel de pensamiento crítico en los estudiantes. Los resultados obtenidos al compararlos con lo planteado por Paul y Elder (2004) que señalan que quien se ejercita en el pensamiento crítico: tiene agudeza perceptiva, hace cuestionamientos permanentes, construye y reconstruye saberes, es de mente abierta, posee una valoración justa, tiene control emotivo y coraje intelectual. Tienen más oportunidades en cualquier campo que elija o su ámbito profesional.

Asimismo, los estudiantes son capaces de defender y justificar sus valores intelectuales y personales, ofrecer y criticar argumentos, apreciar el punto de vista de los 
ESTRATEGIAS COGNITIVAS PARA EL FORTALECIMIENTO DEL PENSAMIENTO CRÍTICO EN ESTUDIANTES DE EDUCACIÓN MENCIÓN CIENCIAS SOCIALES

Edith Gouveia, Maxula Atencio y Janeth de Nobrega (p. 28-51)

demás; incentiva a construir su propio conocimiento, orientado al logro de una comprensión profunda y a su vez significativa del contenido de aprendizaje, incide de manera positiva en el manejo de una serie de capacidades subordinadas, desalienta el tipo de aprendizaje en el que el estudiante es un elemento pasivo de esta manera se prepara individuos para que gocen de una vida productiva, responsable y armoniosa.

Por otro lado, Fernández, (2009), menciona que las operaciones mentales deben ser guiadas por el docente de una forma constante y continua, ya que los estudiantes necesitan aprender a organizar, codificar, procesar, comprender los contenidos o la información para que sea utilizada en cualquier momento y hasta el futuro de manera coherente, cuanto más oportunidades tenga el estudiante de utilizar la información mayores son las posibilidades de aprender.

\section{REFLEXIONES FINALES}

En cuanto a las descripción de las estrategias cognitivas en estudiantes de la carrera de Educación Mención Ciencias Sociales de la Facultad de Humanidades y Educación de LUZ, se obtuvo un promedio de 2,71 ubicado en la categoría Moderado, indicando que los estudiantes posee moderadamente el manejo de dichas estrategia tales como explorar el material, acceder al conocimiento previo, predecir, formular hipótesis, comparar la nueva información con lo que se sabe, crear imágenes mentales de lo que se está procesando, hacer inferencias, generar preguntas y pedir aclaraciones, seleccionar ideas importantes, elaborar pensando ejemplos, evaluar ideas presentadas en el texto, clasificar información, identificar relaciones y modelos, organizar ideas claves, transferir o aplicar conceptos que usa el individuo para adquirir el conocimiento, lo cual permite afirmar que no existe un manejo total de tales procedimientos; es decir, aquellas características requeridas para fortalecer el pensamiento crítico.

Respecto a caracterizar el pensamiento crítico en estudiantes (Agudeza perceptiva, Cuestionamiento, Construcción y reconstrucción del saber, Mente abierta, Coraje intelectual, Autorregulación, Control emotivo, Valoración Justa) los resultados obtenidos evidenciaron que los estudiantes en cuestión poseen un dominio de las características del

Revista EDUCARE, Volumen 19, Número 3, Septiembre -Diciembre 2015. ISSN: 2244-7296 Página 48 
pensamiento crítico moderado con un promedio de 2,92.

Es de señalar que el pensamiento crítico supone una actividad creativa, se caracteriza por manejar, dominar las ideas. Su principal función no es generar ideas sino revisarlas, evaluarlas y repasar que es lo que se entiende, se procesa, se comunica mediante los otros tipos de pensamiento (verbal, matemático, lógico).

En cuanto al diseño de estrategias cognitivas para el fortalecimiento del pensamiento consiste en ayudar a mejorar la habilidad de pensar, no obstante hay que señalar que es complicado, especialmente cuando a los estudiantes se les ha enseñado de forma memorística y que exista una resistencia al cambio por parte de ellos y el docente, ambos deben estar comprometidos con la tarea de desarrollar clases dinámicas, interactivas. Las estrategias sirven de activadoras para que los docentes inventen los suyos o generen las adaptaciones necesarias. Lo importante es que los docentes se orienten en esencia a desarrollar procesos y crear estructuras lógicas de adquisición de capacidades y conocimientos en los estudiantes.

\section{REFERENCIAS}

Araujo, F. (2011). Estrategias pedagógicas para fomentar el pensamiento crítico en los estudiantes de la escuela de ciencia política. Trabajo de grado para optar al título de Magíster en Educación mención Currículo. Universidad del Zulia. Facultad de Humanidades y Educación

Beltrán, J. (1996) Procesos, estrategias y técnicas de aprendizaje. Madrid: Editorial Síntesis, S.A.

Chadwick y Rivera (1991). Estrategias Cognitivas. Metacognitivas y el uso de los Microcomputadores en la Educación. PLANIOC.

Chávez, N. (1994). Introducción a la Investigación científica. Maracaibo. Universidad del Zulia

Delors, J (1996). La educación encierra un tesoro: informe de la Comisión Internacional de la educación para el siglo XXI. UNESCO. Madrid: Santillana

Elliott, J. (1993) El cambio educativo desde la investigación-acción, Madrid: Morata.

Fernández, M. (2009). Estrategias de enseñanza para el logro del pensamiento crítico en el

Revista EDUCARE, Volumen 19, Número 3, Septiembre -Diciembre 2015. ISSN: 2244-7296 Página 49 
aprendizaje de la geografía. Trabajo de grado para optar al título de Magíster en Geografía, mención Docencia. Universidad del Zulia. Facultad de Humanidades y Educación.

Ennis y Norris (1989) Evaluatingcriticalthinking. Critical thinking press \& software. Pacific Grove.

Galindo, Y. (2010). Estrategias cognoscitivas para la enseñanza y aprendizaje de la nomenclatura y formulación de compuestos inorgánicos. Trabajo de grado para optar al título de Magister Scientiarum en Enseñanza de la Química. Universidad del Zulia. Facultad de Humanidades y Educación

Gaskins y Elliot (1999). Cómo enseñar estrategias cognitivas en la escuela: el manual Benchmark para docente. Editorial Paidós. Mexico

Hartman, H. y Sternberg, R. (1993). A broad BACEIS for improving thinking. InstructionalScience, 21, 401-425.

Ley Orgánica de Educación. (2009). Gaceta Oficial Extraordinario No. 5.929. Consulta: 15 de diciembre de 2012.

Paredes, A. (2006). "Creatividad: Estrategias de Desarrollo”. Universidad Peruana Unión. Lima, Perú.

Paúl R. y Elder L.. (2004). Una guía para los educadores en los estándares de competencia para el pensamiento crítico. Nueva York.

Pírela, N. (2011). Estilos de pensamiento y estrategias de aprendizaje utilizadas por los estudiantes de la asignatura geografía general. Trabajo de grado para optar al título de Magíster en Geografía, mención Docencia. Universidad del Zulia. Facultad de Humanidades y Educación.

Rigney, J.. (1978). Learning strategies: a theoretical perspective. En O’Neil, H.F. (Ed.): Learning strategies. Academic Press. USA.

Romero, I. (2012). Seminario investigativo como estrategia didáctica para desarrollar pensamiento crítico en la enseñanza de la geografía. Trabajo de grado para optar al título de Magíster en Geografía, mención Docencia. Universidad del Zulia. Facultad de Humanidades y Educación.

Sánchez, L. (1997) Las Estrategias Cognitivas y el Aprendizaje. Ediciones Morata. Madrid.

UNESCO. (Organización de las Naciones Unidas para la Educación, la Ciencia y la Cultura). (1998). Hacia un programa para la Educación Superior. Documento presentado en la Conferencia Mundial sobre la Educación Superior: La Educación Superior en el siglo XXI. Visión y Acción Paris.

Revista EDUCARE, Volumen 19, Número 3, Septiembre -Diciembre 2015. ISSN: 2244-7296 Página 50 
Zapata, M. (2005) Secuenciación de contenidos y objetos de aprendizaje. RED. Revista de Educación a Distancia, número monográfico II. http://www.um.es/ead/red/M2/zapata47.pdf Revisado el 12/12/2012. 